\title{
The sampling distributions of Gaussian ROC statistics
}

\author{
NEIL A. MACMILLAN and CAREN M. ROTELLO \\ University of Massachusetts, Amherst, Massachusetts \\ and \\ JEFF O. MILLER \\ University of Otago, Dunedin, New Zealand
}

\begin{abstract}
For a discrimination experiment, a plot of the hit rate against the false-alarm rate- the ROC curvesummarizes performance across a range of confidence levels. In many content areas, ROCs are well described by a normal-distribution model and the $z$-transformed hit and false-alarm rates are approximately linearly related. We examined the sampling distributions of three parameters of this model when applied to a ratings procedure: the area under the ROC $\left(A_{z}\right)$, the normalized difference between the means of the underlying signal and noise distributions $\left(d_{a}\right)$, and the slope of the ROC on $z$-coordinates $(s)$. Statistical bias (the degree to which the mean of the sampling distribution differed from the true value) was trivial for $A_{z}$, small but noticeable for $d_{a}$, and substantial for $s$. Variability of the sampling distributions decreased with the number of trials and was also affected by the number of response categories available to the participant and by the overall sensitivity level. Figures in the article and tables available on line can be used to construct confidence intervals around ROC statistics and to test statistical hypotheses.
\end{abstract}

Signal detection theory (SDT; Green \& Swets, 1966) offers investigators a tool for separating sensitivity from bias effects, comparing performance across paradigms, and predicting accuracy quantitatively from aspects of the stimulus array. SDT is, accordingly, widely used to analyze data from behavioral experiments. The indices of performance prescribed by SDT, like all statistics, are estimates of theoretical parameters, and the quality of the estimates affects substantive conclusions.

Every performance index has a sampling distribution, which is the frequency distribution of possible values that can occur for a particular true value of the parameter. The two most important properties of a sampling distribution are its mean and its variance. If the mean equals the population parameter, the index is said to be unbiased. An unbiased estimator equals, on the average, the parameter it estimates, and such common measures as the sample mean and observed proportion have this characteristic. The second property is the distribution's variance; low variance (high efficiency) is desirable. We also use the terms accuracy and precision, respectively, to refer to the degrees of bias and of efficiency.

\footnotetext{
This research was supported in part by National Institutes of Health Research Grant MH60274 to C.M.R. and N.A.M. We thank Jerry Balakrishnan, Michael Hautus, Lawrence Ward, and an anonymous reviewer for helpful and/or provocative comments on an earlier draft. Correspondence concerning this article should be addressed to N. A. Macmillan, Department of Psychology, Tobin Hall, University of Massachusetts, Amherst, MA 01003 (e-mail: nam@psych.umass.edu).
}

Miller (1996) calculated the sampling distribution of the popular sensitivity index $d^{\prime}$. He examined a range of values for true $d^{\prime}$ and the number of trials per stimulus and considered three conventions for dealing with cases in which $d^{\prime}$ is undefined (because proportions of 0 or 1 occur). For each choice of these parameters, the complete sampling distribution was calculated from the binomial distributions of hits and false alarms.

One unsurprising result of the bias calculation was that estimates were increasingly accurate as the number of trials increased. With smaller data sets, either positive or negative bias could occur. The most problematic cases were those in which true sensitivity was high, because these are the situations for which the correction for zero and one cells is most needed, and any correction leads to some distortion in the estimate. This pattern of findings might affect substantive conclusions in (at least) two ways: First, comparisons of sensitivity values involving different numbers of trials entail a constant error, so that true differences in sensitivity might be greater or less than obtained ones. Experimenters typically use equal numbers of trials in conditions to be compared, avoiding this problem by design, but the second threat is more insidious: Comparisons of different sensitivity values based on the same number of trials also introduce an error.

For the sampling distribution variance, a larger number of trials always leads to a smaller variance and, thus, to a more precise approximation. Quantitatively, the variances of many statistics (again including the sample mean and observed proportion) are proportional to $1 / N$, but the pat- 
tern for $d^{\prime}$ was much less regular, particularly at high sensitivity levels. In this respect, Miller's (1996) computations improved on the earlier approximation of Gourevitch and Galanter (1967), which incorrectly displays greater regularity and tends to underestimate the true variance, especially for $N_{\text {trials }}<100$ or even moderately high values of $d^{\prime}$.

Miller's (1996) results apply to the one-interval yes-no design, in which a sample of either a signal (S) or noise (N) is presented on each trial and the observer makes a binary response. The statistic $d^{\prime}$ can also be estimated from more complex experimental paradigms, each with a different decision rule and sampling distributions. Calculations have been performed for two- and three-interval forced choice, three-choice oddity, and four-interval same-different tasks (Bi, Ennis, \& O’Mahony, 1997) and for two-interval same-different designs (Bi, 2002). In some cases, only one of several possible models for these designs has been evaluated.

\section{Rating Experiments and ROC Curves}

SDT models for the yes-no experiment assume that each stimulus class leads to a distribution of values, and represent sensitivity as the difference between the means of these two distributions. The most common assumption about the form of the distributions, and the one that we make in this article, is normality; Swets (1986a, 1986b) showed that data across a wide range of content areas were consistent with this assumption. To calculate $d^{\prime}$, however, is to make the stronger assumption that the two distributions have the same variance: $d^{\prime}$ equals the mean difference divided by the common standard deviation $(S D)$. This assumption is much less often supported, as Swets (1986a, 1986b) also showed.

Support for a Gaussian model with unequal variances comes from receiver operating characteristic (ROC) curves, which are most often generated in confidencerating designs. ROC curves plot the hit rate $[P($ "yes"| $\mid \mathrm{S})]$ versus the false-alarm rate $[(P($ "yes" $\mid \mathrm{N})]$ for the various levels of confidence; a sample ROC is shown in Figure 1. Having several ROC points rather than the single point provided by a yes-no design allows the estimation of two sensitivity parameters rather than one and permits goodnessof-fit evaluation of the Gaussian model. ${ }^{1}$

One of the two sensitivity parameters, analogous to $d^{\prime}$, is the difference between the means divided by some kind of $S D$. Several proposals have been made about the appropriate divisor; we adopt the root-mean-square average of the $\mathrm{S} S D$ (which we set equal to 1) and the N $S D$ (denoted $s$ ). This measure of sensitivity is

$$
d_{a}=\left(\mu_{\mathrm{s}}-\mu_{\mathrm{n}}\right) /\left(1+s^{2}\right)^{1 / 2},
$$

where $\mu_{\mathrm{s}}$ and $\mu_{\mathrm{n}}$ are the means of the $\mathrm{S}$ and $\mathrm{N}$ distributions. In ROC space, higher values of the index $d_{a}$ indicate that the ROC is farther from the major diagonal. If the hit and false-alarm rates are converted to $z$-scores to produce a ${ }_{z} \mathrm{ROC}$ (Figure 1B), $d_{a}$ corresponds to the distance between the ROC and the major diagonal at a particular
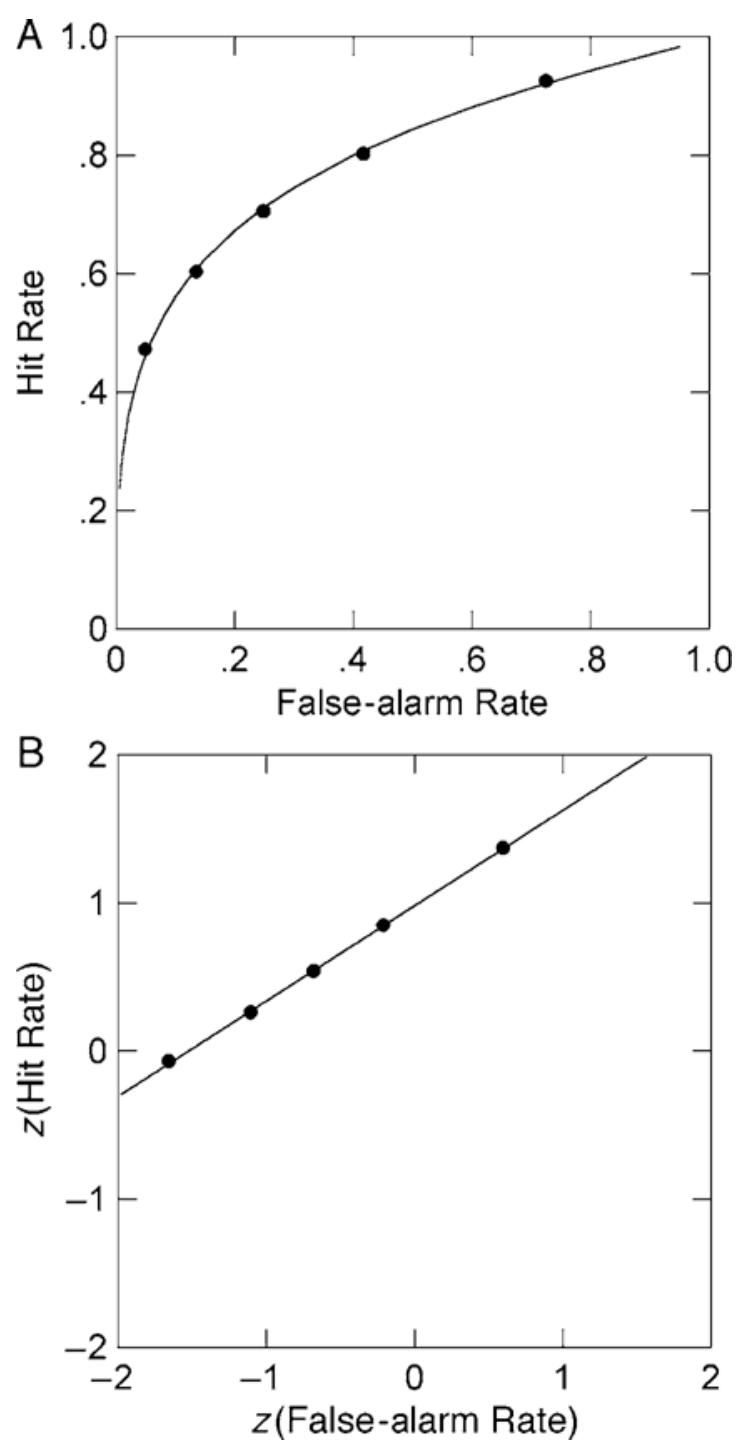

Figure 1. Normal-distribution receiver operating characteristic curves in (A) linear coordinates and (B) $z$-coordinates after Rotello, Macmillan, and Van Tassel (2000, Experiment 1, Figure 3).

point (see Macmillan \& Creelman, 1991, for more detail). A second sensitivity statistic that is equivalent to (i.e., a monotonic transformation of) $d_{a}$ is $A_{z}$, the area under the normal-normal ROC. The relation between the two parameters is

$$
A_{z}=\Phi\left(d_{a} / \sqrt{2}\right)
$$

where $\Phi$ is the cumulative normal distribution function.

The second measure that can be estimated from ROC data is simply $s$, the ratio of the $\mathrm{N}$ and $\mathrm{S} S D$ s. (In our notation, $s$ also equals the N $S D$ itself, because we set the $\mathrm{S}$ $S D$ to 1 , but this is not necessary.) The index $s$ is the slope of the $z$ ROC curve. The rationale for studying $s$ may be less obvious than that for examining $d_{a}$, but $s$ has been used to test theoretical models in areas ranging 
from sensory psychology to recognition memory. For example, certain classes of recognition memory models predict that $s$ will decrease as the memory strength of encoded items increases, and whether this generalization holds has been a matter of controversy (Glanzer, Kim, Hilford, \& Adams, 1999; Ratcliff, McKoon, \& Tindall, 1994).

There have been a few previous discussions of the statistics of ROC parameters. Pollack and Hsieh (1969) calculated the bias and standard error of $A_{g}$ (an area measure found by simply "connecting the dots" in an ROC plot) and a transformation of $d_{e}^{\prime}$ (a distance measure equal to the difference between the means of the underlying distributions divided by the average of their $S D$ s). Although these indices are not exactly equivalent to $A_{z}$ and $d_{a}$, they bear a family resemblance, and we shall see that our results for the most part mirror Pollack and Hsieh's. Swets and Pickett (1982) showed how to use real data to estimate the standard error of $A_{z}$ and partition it among within-observer, within-stimulus, and noise components. To our knowledge, no one has examined the statistical properties of $s$.

\section{PRESENT CALCULATIONS}

We set out to estimate the accuracy and precision of $A_{z}$, $d_{a}$, and $s$ by finding the means and $S D$ s of their sampling distributions. Unlike $d^{\prime}$, which can be calculated directly from hit and false-alarm rates, ROC statistics must be derived from a procedure that fits the best ROC curve to the data points. We used ROCKIT, Metz's (1998) implementation of Dorfman and Alf's (1969) maximum likelihood estimation procedure. ROCKIT accepts as input a stimulus-response matrix from a rating experiment. It assumes that the true ROC is a straight line on $z$-axes (or, equivalently, that the underlying distributions are normal after an unknown monotonic transformation) and calculates the parameters of the ROC. Maximum likelihood is preferred over regression because both ROC values - the hit rate and the false-alarm rate - are variable.

The simulation method followed from the representation assumed by SDT, which is illustrated in Figure 2 . The observer in a rating task with $N_{\text {resps }}$ possible responses divides a continuum into $N_{\text {resps }}$ regions by the use of $\left(N_{\text {resps }}-1\right)$ criteria. The criteria were assumed to be fixed across trials, and their placements varied across simulation conditions, as we will describe later. For each of $N_{\mathrm{s}}$ simulated signal trials and $N_{\mathrm{n}}$ simulated noise trials, a random number was generated from the corresponding (signal or noise) normal distribution using the Box-Muller method (Devroye, 1986). The location of this random number relative to the fixed response criteria determined the confidence rating response for that simulated trial. The distributions of such responses were tabulated across all $N_{\mathrm{s}}$ simulated signal trials and across all $N_{\mathrm{n}}$ simulated noise trials (as they would be for a human observer). The resulting stimulus-response matrix for each simulated observer was submitted to ROCKIT,

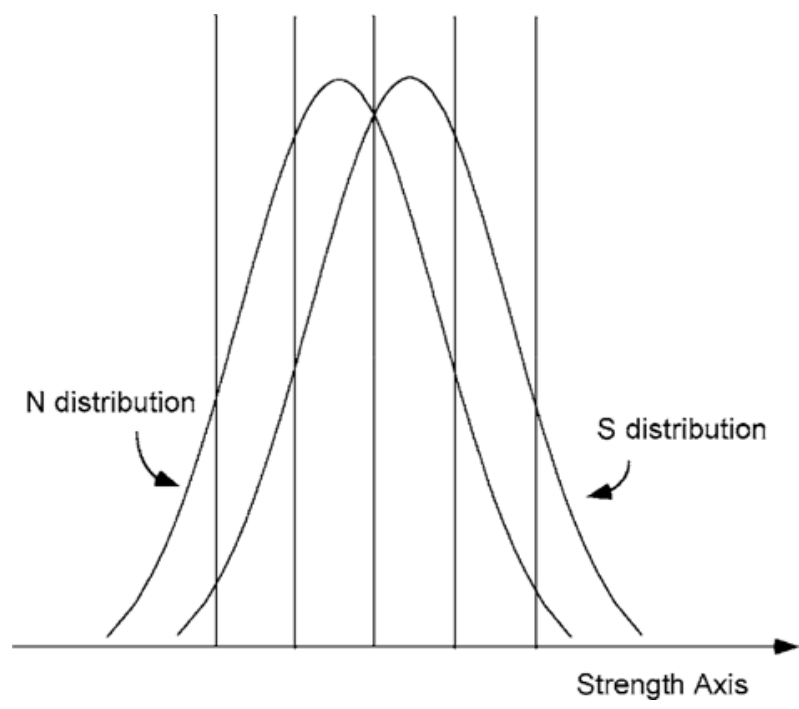

Figure 2. Underlying representation for the rating experiment.

which obtained the maximum-likelihood estimates of $d_{2}^{\prime}$ (the distance between the signal and noise distributions in units of the signal $S D$, denoted $A$ in Metz's, 1998, program) and $s$ (denoted $B$ in the program). The estimated value of $d_{a}$ was then obtained using the transformation

$$
d_{a}=\left[2 /\left(1+s^{2}\right)\right]^{1 / 2} \cdot d_{2}^{\prime} .
$$

The estimated value of $A_{z}$, which is also given by the program, is related to $d_{a}$ by Equation 2 . We simulated 1,000 observers for each of a variety of different parameter values and then calculated the mean and $S D$ of each estimated value across the 1,000 simulated observers.

Each set of simulations was based on a particular choice of five variables: true $d_{a}$, true $s, N_{\text {trials }}$ (which equaled both $N_{\mathrm{s}}$ and $N_{\mathrm{n}}$ ), $N_{\text {resps }}$, and criterion placement. We considered three possible criterion placements: (1) a symmetric arrangement (as in Figure 2), with the same area critp falling below the lowest criterion under the $\mathrm{N}$ distribution and above the highest criterion under the $\mathrm{S}$ distribution; (2) a conservative response bias, with criteria shifted toward higher values by allowing the lowest criterion to cut off an area of 2 times critp under the $\mathrm{N}$ distribution, whereas the highest criterion cut off an area of critp under the S distribution; and (3) a liberal response bias, with criteria shifted toward lower values by allowing the highest criterion to cut off an area of 2 times critp under the $\mathrm{S}$ distribution, whereas the lowest criterion had an area of critp under the $\mathrm{N}$ distribution (the correct rejection rate). In all cases, the intermediate criteria were evenly spaced. In the research areas surveyed by Swets (1986a), symmetrical placement (the lowest criterion as far below the $\mathrm{N}$ mean as the highest was above the $\mathrm{S}$ mean, within $0.5 z$-units) was obtained for visual detection by humans ( 2 of 4 observers) and pigeons. A conservative shift (the lowest criterion more than $0.5 z$-units lower relative to the $\mathrm{N}$ mean than the highest was above the $\mathrm{S}$ mean) held 
for visual detection ( 2 of 4 observers), screening of slides, and information screening. A liberal shift was observed for recognition memory of words, recognition memory of odors, and medical diagnosis. Spacing of criteria between the extremes was generally even. It is not likely that this pattern of criterion placements uniquely characterizes content areas; we conclude only that all these patterns do occur.

Parameter values used in the simulations are given in Table 1. Like Miller (1996), we encountered cases in which estimates of the parameters could not be obtained. Metz's (1998) program rejects degenerate data sets in which the signal detection parameters cannot be estimated uniquely [e.g., because of perfect decision performance, only a single operating point other than $(0,0)$ and $(1,1)$, etc.]. For each set of 1,000 simulations, we counted the number of times the fitting procedure did not converge, and if this was greater than 20 we did not try to summarize the data. (This decision reflects our belief that researchers faced with such data sets in practice would have no choice but to discard them as "pathological," and would most likely repeat the experiment with conditions altered to yield nonpathological results.) The offending cases (as in Miller, 1996) tended to be those in which true $d_{a}$ was high and/or $N_{\text {trials }}$ was low.

\section{RESULTS}

We calculated bias (average observed value minus true value) and standard error for $A_{z}, d_{a}$, and $s$. For each case, we simulated both symmetric and asymmetric criteria, as described earlier, yielding 18 sets of calculations. Within each of these, we examined the effects of two parameters that are under direct experimenter control $\left(N_{\text {trials }}\right.$ and $\left.N_{\text {resps }}\right)$ and three that are not (true $d_{a}$, true $s$, and critp).

Seeking an overview of the dependencies among these statistics, we conducted regression analyses on each set of simulations. We regressed the log of each dependent variable (bias and standard error of $A_{z}, d_{a}$, and $s$ ) on all of the parameters under investigation, their squares, and their products. For the bias calculations, the regressions gave little information about the observed data patterns, and we do not describe these results. The outcome of the standard error calculations is summarized in Table 2. (Only variables contributing at least $5 \%$ of the variance are included in the table.) The variable $N_{\text {trials }}$ is the first or second most predictive variable, as a main effect or as part of an interaction, in each regression. Its coefficient

Table 1

Values of Parameters Used in Simulations

\begin{tabular}{ll}
\hline Parameter & \multicolumn{1}{c}{ Possible Values } \\
\hline true $d_{a}$ & $0-5$ in steps of 0.25 \\
true $s$ & $0.25,0.5-1.5$ in steps of $0.1,1.75,2.0,2.5,3.0,4.0$ \\
$N_{\text {trials }}$ & $50,100,200,500,1,000$ \\
$N_{\text {resps }}$ & $4,5,6,7,8,10$ \\
critp & $.05, .10, .15, .25$ \\
\hline
\end{tabular}

is usually negative, as would be expected from the typical dependence of standard errors on sample size. True $d_{a}$ is also the first or second most predictive variable, as a main effect or as part of an interaction, in each regression for $S D\left(A_{z}\right)$ and $S D\left(d_{a}\right)$. Its coefficient is usually positive, as would be expected from the increasing standard error of $z$ with discrimination accuracy. True $s$ is the most predictive variable in two of the regressions for $S D(s)$ and is the third most predictive variable in the other. Its coefficient is always positive. These results are sensible, but because the range of the variables is so large they do not provide adequate guidance for specific cases.

In this section, we summarize the results of the calculations in a series of graphs, one multipanel figure for each combination of SDT statistic $\left(A_{z}, d_{a}\right.$, and $\left.s\right)$ and type of assessment (accuracy and precision). Each graph includes symmetric, conservative, and liberal criterion placement assumptions. We averaged across the four possible values of critp; in general, both statistical bias and standard error increased with critp, but most of this effect arose from the critp $=.25$ case. Thus, the values in the figures slightly overestimate the true values for critp $=.05, .10$, and .15 , and noticeably underestimate them for critp $=.25$. All values of true $s$ and most values of true $d_{a}(0-5$ in steps of .5) are considered, but for only three sets of the experimenter-controlled variables $\left(N_{\text {trials }}=50, N_{\text {resps }}=6 ; N_{\text {trials }}=200, N_{\text {resps }}=6\right.$; and $N_{\text {trials }}=50, N_{\text {resps }}=10$ ). These values span typical choices by experimenters and allow for simple comparisons of conditions differing only in $N_{\text {trials }}$ or only in $N_{\text {resps }}$. Trends in the full data set are largely captured by this subset.

A listing that gives the results for all combinations of the variables can be found at http://www-unix.oit.umass.edu/ $\sim$ caren/Design/Assets/index.htm. Visitors to this site first select the criterion parameters (value of critp and symmetric, liberal, or conservative placement) most appropriate for their data set, and then choose tables giving statistical bias and standard error as a function of the experimenter variables $N_{\text {trials }}$ and $N_{\text {resps }}$ and the observer parameters true $d_{a}$ and true $s$.

\section{Area Sensitivity Measure $\boldsymbol{A}_{z}$}

Accuracy (statistical bias). Figure 3 gives the statistical bias (i.e., the average value observed in our simulations minus the true value) associated with estimating $A_{z}$. Values plotted are percents of $A_{z}$. The most striking result is that the bias is very low, ranging only up to about $3 \%$ for symmetric placement of the criteria and about $1 \%$ for asymmetric placement. Increasing $N_{\text {trials }}$ decreases what bias there is; changing $N_{\text {resps }}$ has little effect. True sensitivity and slope have small and unsystematic effects, trivial in the range of slopes $(0.5<s<1.5)$ that is typical.

These findings can be compared with those of Pollack and Hsieh (1969). Their method differed from ours in that $N_{\text {trials }}$ and $N_{\text {resps }}$ were not independently determined. Instead, a number of samples, $n$, of both the $\mathrm{S}$ and the $\mathrm{N}$ distributions were chosen, and an ROC was constructed 
Table 2

Results of Regression Analyses of (Log-Transformed) Standard Errors of Estimated $A_{z}, d_{a}$, and $s$

\begin{tabular}{|c|c|c|c|c|c|c|c|c|c|}
\hline \multirow{3}{*}{$\begin{array}{l}\text { Dependent } \\
\text { Variable }\end{array}$} & \multicolumn{9}{|c|}{ Criterion Placements } \\
\hline & \multicolumn{3}{|c|}{ Symmetric } & \multicolumn{3}{|c|}{ Conservative } & \multicolumn{3}{|c|}{ Liberal } \\
\hline & Variable & $\beta$ & $R^{2}$ & Variable & $\beta$ & $R^{2}$ & Variable & $\beta$ & $R^{2}$ \\
\hline $\ln \left(S D A_{z}\right)$ & $\begin{array}{l}\text { Total } \\
d_{a} \times N_{\text {trials }} \\
N_{\text {trials }}\end{array}$ & $\begin{array}{l}-.00009 \\
-.0036\end{array}$ & $\begin{array}{l}.70 \\
.53 \\
.06\end{array}$ & $\begin{array}{l}\text { Total } \\
N_{\text {trials }} \times \text { critp } \\
d_{a} \times \text { critp } \\
N_{\text {trials }^{2}}{ }^{2}\end{array}$ & $\begin{array}{l}-.0027 \\
-.30 \\
+.000002\end{array}$ & $\begin{array}{l}.89 \\
.66 \\
.12 \\
.06\end{array}$ & $\begin{array}{l}\text { Total } \\
N_{\text {resps }} \times \text { critp } \\
N_{\text {trials }} \times d_{a} \\
d_{a} \times \text { critp } \\
N_{\text {trials }} \\
d_{a}{ }^{2}\end{array}$ & $\begin{array}{l}+.0065 \\
+.000147 \\
+3.71 \\
-.00149 \\
-.239\end{array}$ & $\begin{array}{l}.77 \\
.24 \\
.20 \\
.11 \\
.06 \\
.05\end{array}$ \\
\hline $\ln \left(S D d_{a}\right)$ & $\begin{array}{l}\text { Total } \\
N_{\text {trials }} \\
d_{a}^{2} \\
N_{\text {trials }}^{2}\end{array}$ & $\begin{array}{l}-.0035 \\
+.081 \\
+.000002\end{array}$ & $\begin{array}{l}.83 \\
.46 \\
.28 \\
.08\end{array}$ & $\begin{array}{l}\text { Total } \\
N_{\text {trials }} \times \text { critp } \\
d_{a}^{2} \\
N_{\text {trials }^{2}}^{2}\end{array}$ & $\begin{array}{l}-.0035 \\
+.13 \\
+.000002\end{array}$ & $\begin{array}{l}.93 \\
.53 \\
.25 \\
.08\end{array}$ & $\begin{array}{l}\text { Total } \\
d_{a} \times \text { critp } \\
N_{\text {trials }} \\
d_{a}^{2} \\
N_{\text {resps }} \times d_{a}\end{array}$ & $\begin{array}{l}+1.675 \\
-.00175 \\
-.138 \\
-.0704\end{array}$ & $\begin{array}{l}.67 \\
.30 \\
.12 \\
.07 \\
.07\end{array}$ \\
\hline $\ln (S D s)$ & $\begin{array}{l}\text { Total } \\
s \\
N_{\text {trials }} \\
d_{a}^{2} \\
N_{\text {trials }^{2}}{ }^{2}\end{array}$ & $\begin{array}{l}+1.447 \\
-.004 \\
+.104 \\
+.000002\end{array}$ & $\begin{array}{l}.96 \\
.41 \\
.23 \\
.23 \\
.05\end{array}$ & $\begin{array}{l}\text { Total } \\
s \\
N_{\text {trials }} \times \text { critp } \\
d_{a}^{2} \\
N_{\text {trials }}^{2}\end{array}$ & $\begin{array}{l}+.708 \\
-.0047 \\
+.181 \\
+.000002\end{array}$ & $\begin{array}{l}.96 \\
.33 \\
.28 \\
.19 \\
.08\end{array}$ & $\begin{array}{l}\text { Total } \\
N_{\text {trials }} \\
N_{\text {resps }} \times \text { critp } \\
s \\
N_{\text {trials }} \times d_{a}\end{array}$ & $\begin{array}{l}-.00385 \\
+.474 \\
+.80 \\
+.0004\end{array}$ & $\begin{array}{l}.75 \\
.14 \\
.12 \\
.09 \\
.09\end{array}$ \\
\hline
\end{tabular}

Note-Only variables contributing at least .05 to $R^{2}$ are included. (The independent variables $d_{a}$ and $s$ are true values, not estimated ones.)

by imposing criteria for each value obtained. Thus, if $2 n$ different values were obtained, a $2 n$-point ROC would be obtained; repeated values would decrease the number of points. ${ }^{2}$ The maximum value of $n$ was 40 . This sampling procedure mimics the use of a continuous rating instrument and has the effect of minimizing the quantization error in their connect-the-dots method of calculating area. In comparing our results with Pollack and Hsieh's, the most appropriate parameter values are probably $N_{\text {trials }}=$ $50, N_{\text {resps }}=10$.

Pollack and Hsieh's (1969) Figure 2 displays mean $A_{g}$ as a function of $s^{2}$ and $\left(\mu_{\mathrm{s}}-\mu_{\mathrm{n}}\right) / s$, which we denote $d_{1}^{\prime}$. The true value of $A_{g}$ is the area under the ROC, which equals $A_{z}$ if the distributions are Gaussian. Thus, to obtain an estimate of bias we compare the mean $A_{g}$ they observed with the theoretical value of $A_{z}$ computed from the relation

$$
A_{z}=\Phi\left[s d_{1}^{\prime} /\left(1+s^{2}\right)^{1 / 2}\right],
$$

which can be obtained from Equations 3.1, 3.4, and 3.6 in Macmillan and Creelman (1991). The accuracy of this estimate is within the precision of Pollack and Hsieh's figure.

Precision (standard error). Figure 4 gives the standard error associated with estimating $A_{z}$. For $N_{\text {trials }}=50$, the error is between 0.05 and 0.075 at low levels of sensitivity; the decrease as $N_{\text {trials }}$ increases is approximately with the square root, the rate of convergence found for most statistics. There is no systematic effect of $N_{\text {resps }}$. The size of the error decreases with true $d_{a}$. This is expected, because $A_{z}$ can be viewed as a proportion: It is equal to proportion correct in unbiased two-alternative forced choice (Green \& Swets, 1966). Proportions have binomial variance $p(1-p) / N$, which is smallest for proportions $p$ that are near 0 or 1 . The true slope $s$ has little effect. A summary of the regression for the complete range of variables is given in Table 2 .

Pollack and Hsieh's (1969) calculations again lead to similar results. Because they made no assumptions about ROC shape, the failures of convergence that we encountered did not limit their calculations. Their Figure 5 shows standard errors for $d_{1}^{\prime}$ values as high as $8 \%$; values decrease with $\mathrm{d}_{1}^{\prime}$ and with $s$, falling as low as $0.1 \%$.

The standard error of $A_{z}$ is slightly larger than the binomial $S D$ with $A_{z}$ as parameter. For example, for $N_{\text {trials }}=$ $50, A_{z}=.76$, and $s=1$, the binomial $S D$ is $\left[A_{z}\left(1-A_{z}\right) /\right.$ $100]^{1 / 2}=.043$; in Figure 4, the standard error for this situation $\left(d_{a}=1\right.$ and $\left.s=1\right)$ is about 0.05 . Presumably, the difference is attributable to the use of a rating rather than binary response. The pattern of the precision measurelowest for very high and very low proportions - is like that of the binomial.

Figure 4 contains a few points (especially in the symmetric $N_{\text {trials }}=200, N_{\text {resps }}=6$ case) that are substantially discrepant from the main trends. Examination of such cases shows that they arise from compressed ROC curves. A particular simulation can lead to ROC points that are close together or to ROCs that consist of only 2 or 3 points rather than 5 or more. In those circumstances, the variability of parameter estimates is high. (A similar effect, and a similar explanation, applies to the other ROC statistics.)

\section{Distance Sensitivity Measure $d_{a}$}

Accuracy (statistical bias). Figure 5 gives the bias associated with estimating $d_{a}$, again plotted as a percentage. Overall, the bias is greater than for $A_{z}$. For the symmetric case, it is positive, ranges up to nearly $5 \%$, and tends to decrease with $s$. For the asymmetric cases, the maximum bias is about $3 \%$, with no consistent sign or monotonic trend. For experimenter-controlled vari- 


\section{Asymmetric Criteria}
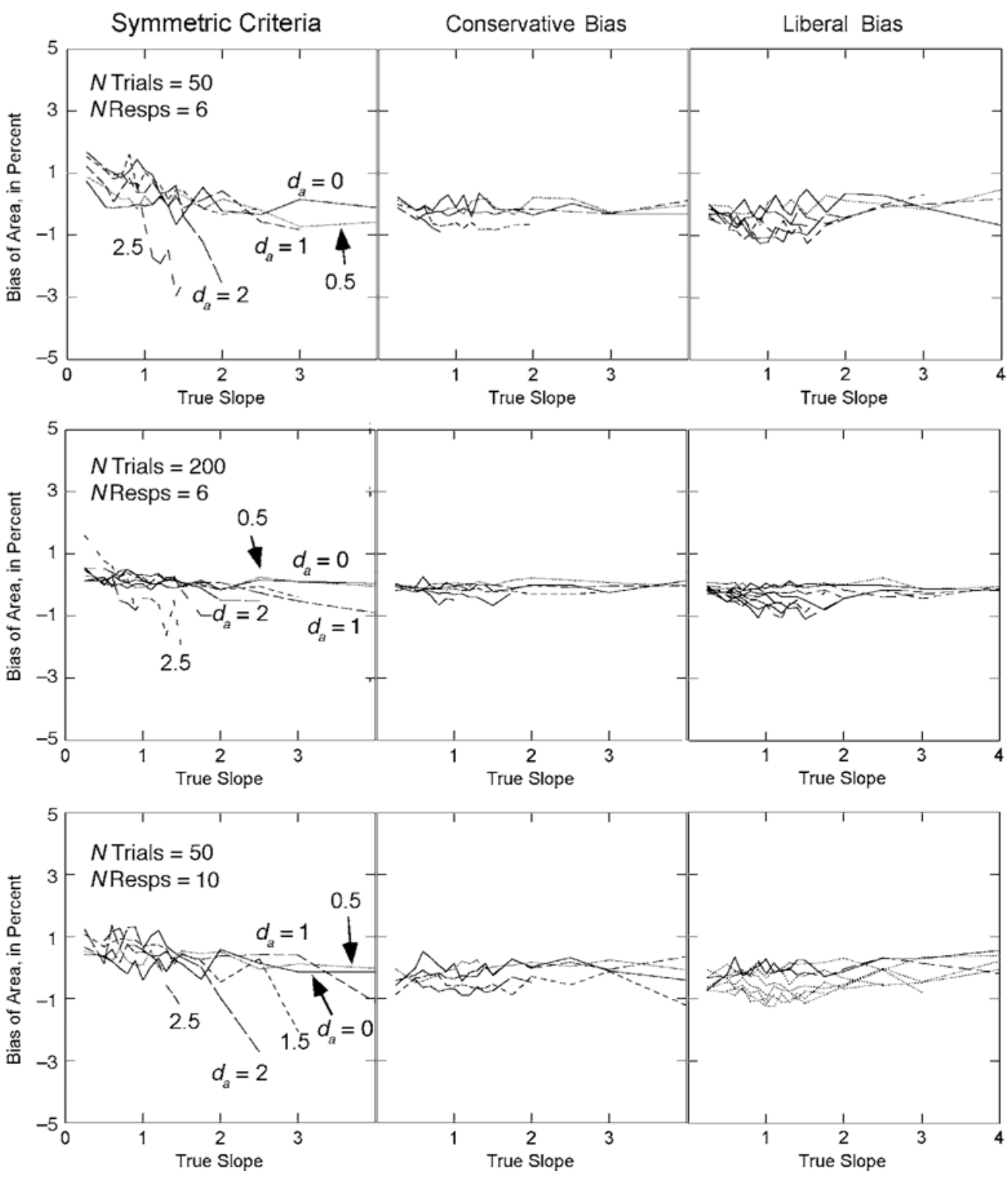

Figure 3. Bias in estimating $A_{z}$, in percent, as a function of true $d_{a}$ and true $s$. Positive values mean that true $A_{z}$ is overestimated. The left panels are for symmetric criterion placement and three different choices of the experimenter-controlled variables $N_{\text {trials }}$ and $N_{\text {resps. }}$. The middle panels are for conservative criterion placement, and the right panels are for liberal criterion placement, all with the same choices of $N_{\text {trials }}$ and $N_{\text {resps }}$.

ables, bias decreases as $N_{\text {trials }}$ increases and is not greatly affected by $N_{\text {resps }}$. Bias does not correlate strongly with sensitivity.

Miller's (1996) results for $d^{\prime}$ were similar. He assumed an unbiased (single) criterion placement, which is presumably most comparable to the symmetric criterion sets in the present study. For $d^{\prime}=1$, bias was $2.9 \%$ for $N_{\text {trials }}=$ $32,0.7 \%$ for $N_{\text {trials }}=128$, and $0.2 \%$ for $N_{\text {trials }}=512$. All biases were about half again as large for $d^{\prime}=2.5$.

Pollack and Hsieh (1969) did not calculate bias for $d_{e}^{\prime}$, but reported the estimated hit rate at the minor diagonal of the ROC as a function of $s^{2}$ and $\mathrm{d}_{1}^{\prime}$. Equations 3.1 and 3.7 from Macmillan and Creelman (1991) reveal that this value should equal $\Phi\left(d_{e}^{\prime} / 2\right)=\Phi\left[d_{1}^{\prime} s /(1+s)\right]$ for underlying normal distributions. Figure 12 of Pollack and Hsieh confirms this prediction, but the scale of the graph is not sufficient to detect biases of the size we observed.

Precision (standard error). Figure 6 gives the standard error associated with estimating $d_{a}$. The error is smaller in the asymmetric cases than in the symmetric case. Error decreases with approximately the square root of $N_{\text {trials }}$; it is largely unaffected by $N_{\text {resps }}$, although in the 

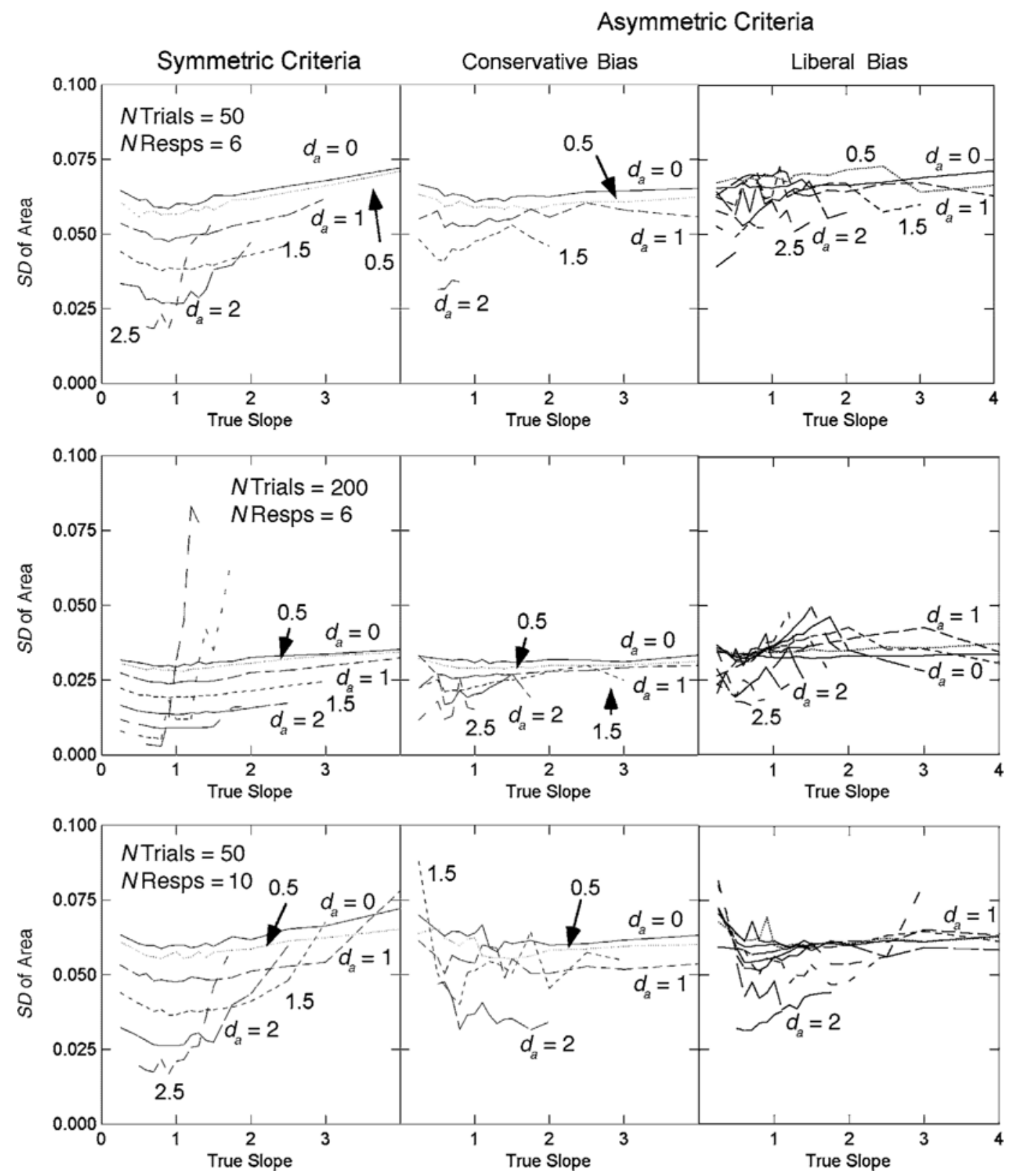

Figure 4. Standard error in estimating $A_{z}$, as a function of true $d_{a}$ and true $s$.

symmetric case some outliers are eliminated. The size of the error is similar for true $d_{a}=0$ and 1 , but increases for $d_{a}$ around 2.5. There is little effect of true $s$. Multiple regression accounted well for this pattern $\left(R^{2}=.94\right.$ for both symmetric and asymmetric placements). Most of the variance in the asymmetric case resulted from two interactions, between true $d_{a}$ and $N_{\text {trials }}$ and between critp and $N_{\text {trials. }}$. A summary of the regression for the complete range of variables is given in Table 2.

The size of the standard error in $d_{a}$ can be compared with that found by Miller (1996) for $d^{\prime}$. For symmetric placement and true $s=1, d_{a}$ is always more precise than $d^{\prime}$, the ratio of the standard errors ranging up to 2 .

\section{SD Ratio (zROC Slope) $s$}

Accuracy (statistical bias). Figure 7 shows that the bias associated with estimating $s$ is noticeably positive, ranging up to $30 \%$. The amount of bias increases with true sensitivity and in most cases with true slope. Increasing $N_{\text {trials }}$ reduces the bias, especially when criterion placements are asymmetric; the effects of $N_{\text {resps }}$ are not systematic. Multiple regression was somewhat successful in accounting 

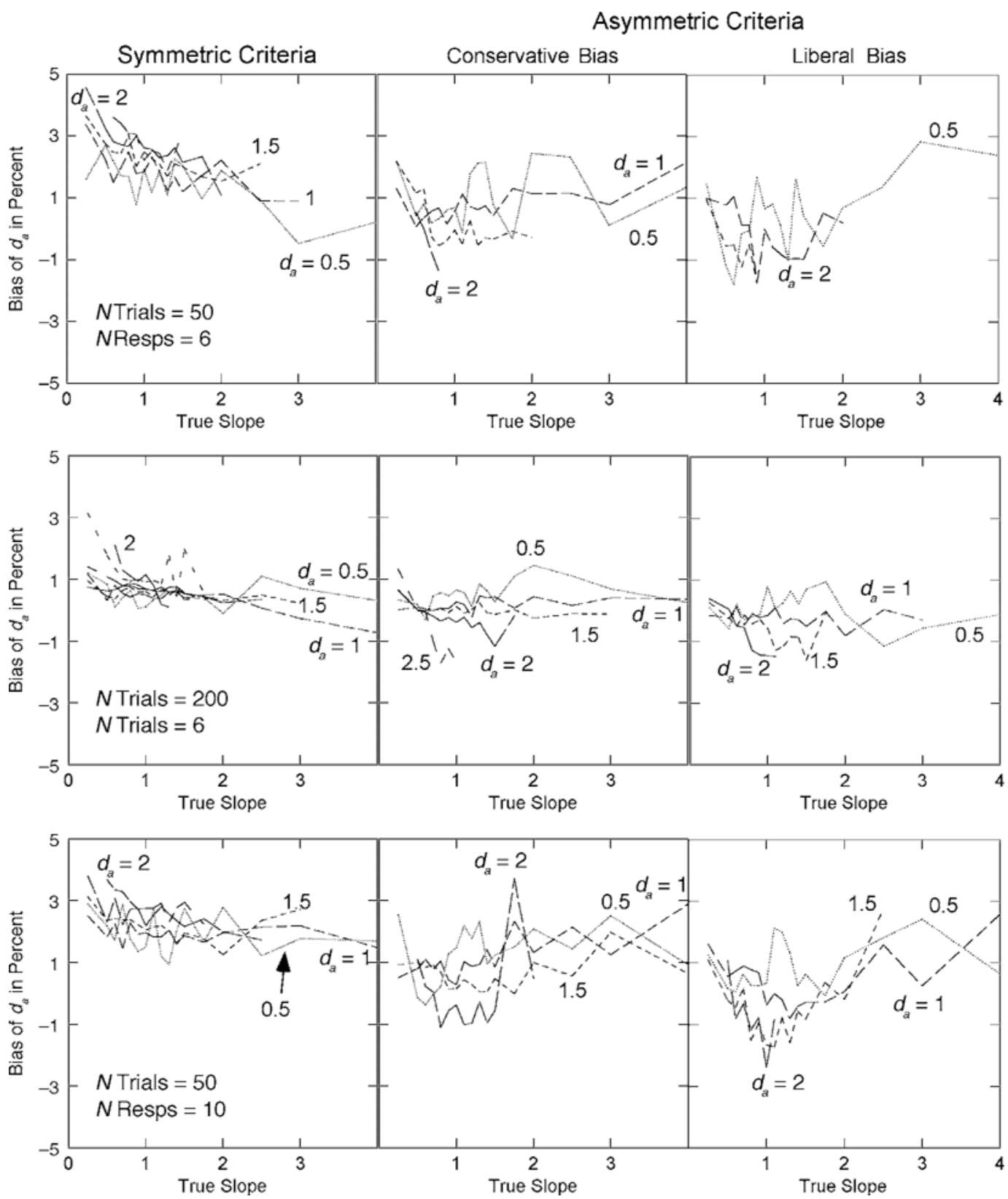

Figure 5. Bias in estimating $d_{a}$, in percent, as a function of true $d_{a}$ and true $s$. Positive values mean that true $d_{a}$ is overestimated.

for this pattern $\left(R^{2}=.77\right.$ for symmetric, .89 for conservative, and .62 for liberal placements). About three quarters of the variance in the conservative case resulted from two interactions, between true $d_{a}$ and true $s$ and between $N_{\text {trials }}$ and critp. About two thirds of the variance in the liberal case resulted from true $s$ and its interaction with $N_{\text {trials. }}$.

Precision (standard error). Figure 8 shows that the standard error associated with estimating $s$ increases with true sensitivity and true slope. The error can be quite large when $N_{\text {trials }}=50$ (up to about $75 \%$ of the magnitude of the slope itself for the cases illustrated in the figure), but decreases with approximately the square root of $N_{\text {trials }}$ (more rapidly in some cases). Even larger errors are evident in Figure 8, especially at high values of true $d_{a}$ and true $s$. Multiple regression (see Table 2) accounted well for this pattern $\left(R^{2}=.94\right.$ for symmetric, .95 for conservative, and .70 for liberal placements). Most of the variance in the asymmetric case resulted from true $d_{a}$, true $s$, and an interaction between critp and $N_{\text {trials. }}$.

\section{Effects of critp}

As was mentioned earlier, the patterns displayed in Figures 3 through 8 are averages across the parameter critp, the area cut off by the most extreme criterion. In 

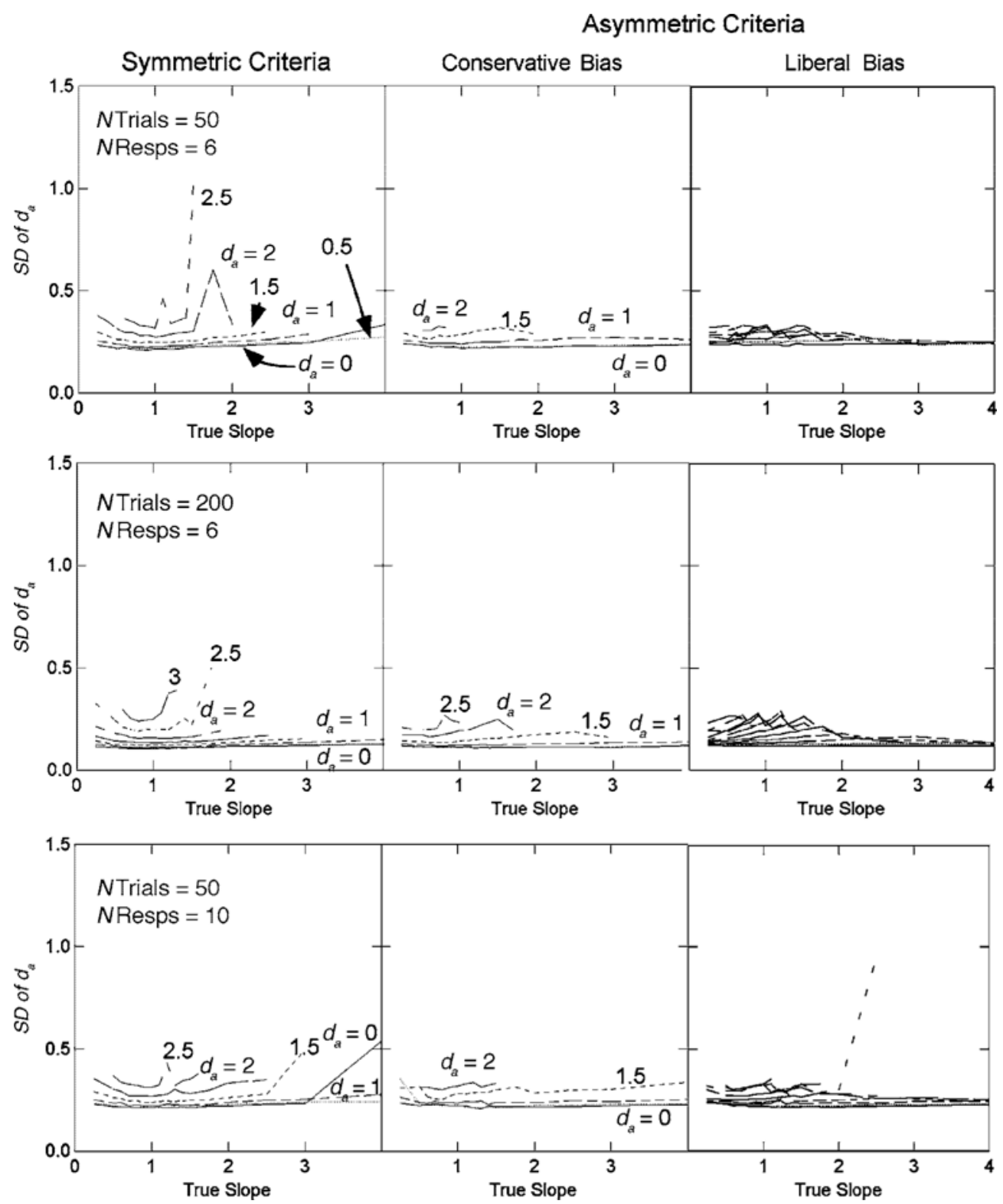

Figure 6. Standard error in estimating $d_{a}$, as a function of true $d_{a}$ and true $s$.

some situations, however, the value can strongly affect characteristics of estimated parameters; this is the case particularly for standard error estimates in the liberal response-bias condition. In Figure 9, the standard errors for each variable for critp $=.05$ and critp $=.25$ are contrasted for the case in which $N_{\text {trials }}=50$ and $N_{\text {resps }}=6$. Standard errors of all three measures are noticeably higher for the critp $=.25$ case. (Note that the scale of $S D$ of area is not the same as in Figure 4.) This pattern is to be expected: If criteria are closely spaced, the slope of the ROC is less constrained than if they are spread far apart, and higher variability results.

The on-line tables do not average across critp, but instead allow the user to choose among values of this parameter.

\section{IMPLICATIONS}

The practical implications of the present calculations fall roughly into the two categories of experimental design and data analysis. We consider each in turn and then provide a brief discussion of the range of applicability of SDT methods.

\section{Experimental Design}

Experimenter-controlled variables. The two variables that are under the experimenter's direct control are the number of trials per stimulus class $\left(N_{\text {trials }}\right)$ and the number of response alternatives available to the participant $\left(N_{\text {resps }}\right)$. To start with the least surprising finding, estimates based on larger numbers of trials are more ac- 

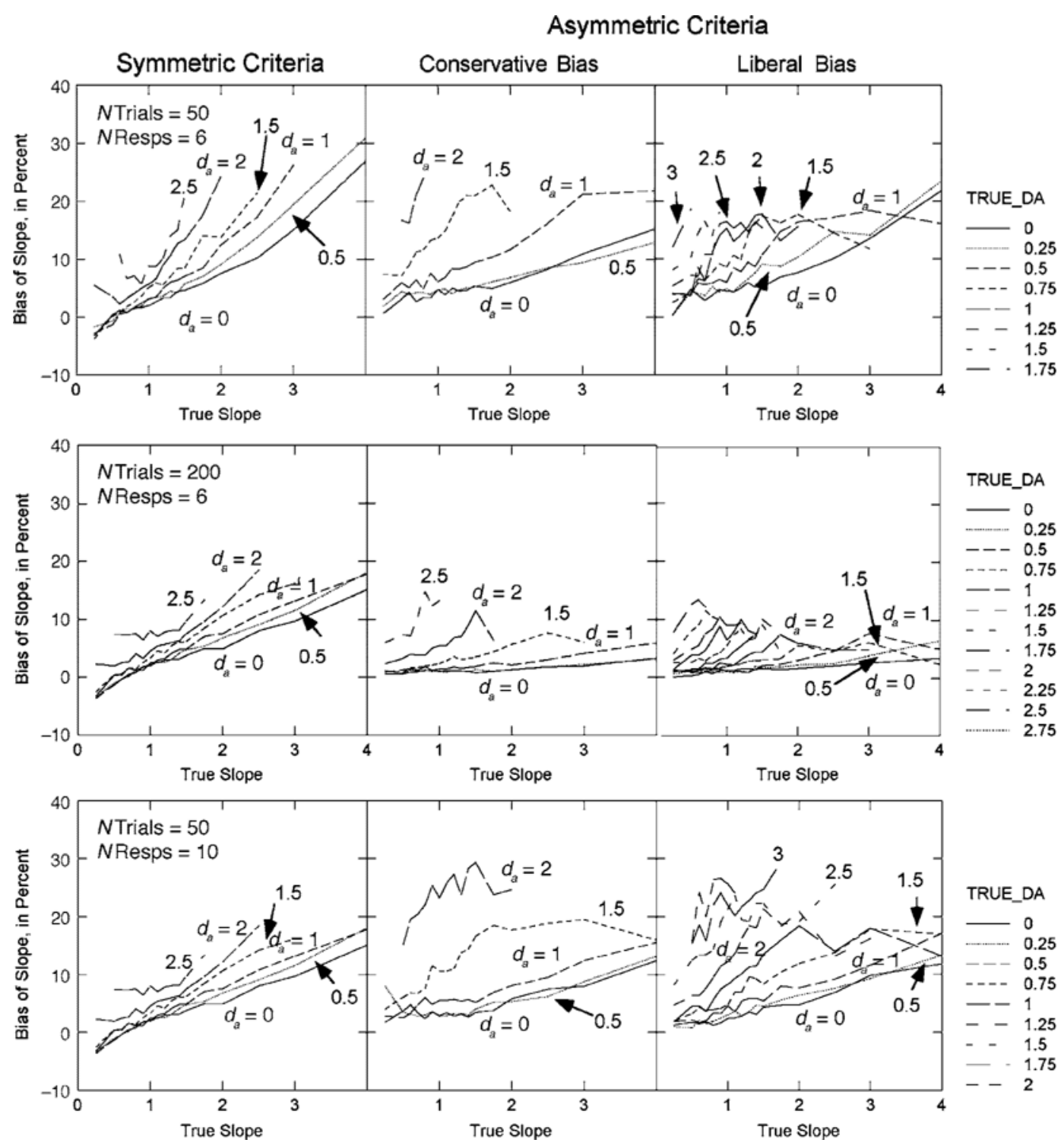

Figure 7. Bias in estimating $s$, in percent, as a function of true $d_{a}$ and true $s$. Positive values mean that true $s$ is overestimated.

curate and precise than those based on fewer trials. For most purposes, 50 trials per stimulus give adequate accuracy and precision for evaluating $A_{z}$ and $d_{a}$, whereas 200 trials are needed for estimates of $s$.

An interesting result that should constrain experimental design is that statistical bias depends on $N_{\text {trials. }}$. The difference between two observed values of $d_{a}$ or $s$ is therefore not a good estimate of the difference between the parameters if the numbers of trials on which the estimates are based differ. In most cases, a constant value of $N_{\text {trials }}$ is natural, but for comparisons among subsets of the data a problem may arise.

Observer characteristics. Two characteristics of observers, true $d_{a}$ and true $s$, have substantial influences on the quality of parameter estimates. The former is usually under indirect control by the experimenter, who can determine whether tasks are easy or difficult. Because of binomial variability, high sensitivity means that proportion measures such as $A_{z}$ are more precise at high $d_{a}$; however, the $z$-transformation reverses this effect so that distance measures such as $d_{a}$ and distance ratios such as $s$ become less precise. Normal-distribution SDT models rely on the $z$-transformation, and fitting them at high levels of sensitivity is problematic. Overall, it appears that keeping true $d_{a}$ below about 2.0 is a desirable goal in experimental planning.

The important take-home design recommendations from the present calculations, therefore, are that (1) the appropriate value of $N_{\text {trials }}$ depends on the parameter being estimated, (2) parameter estimates are best com- 

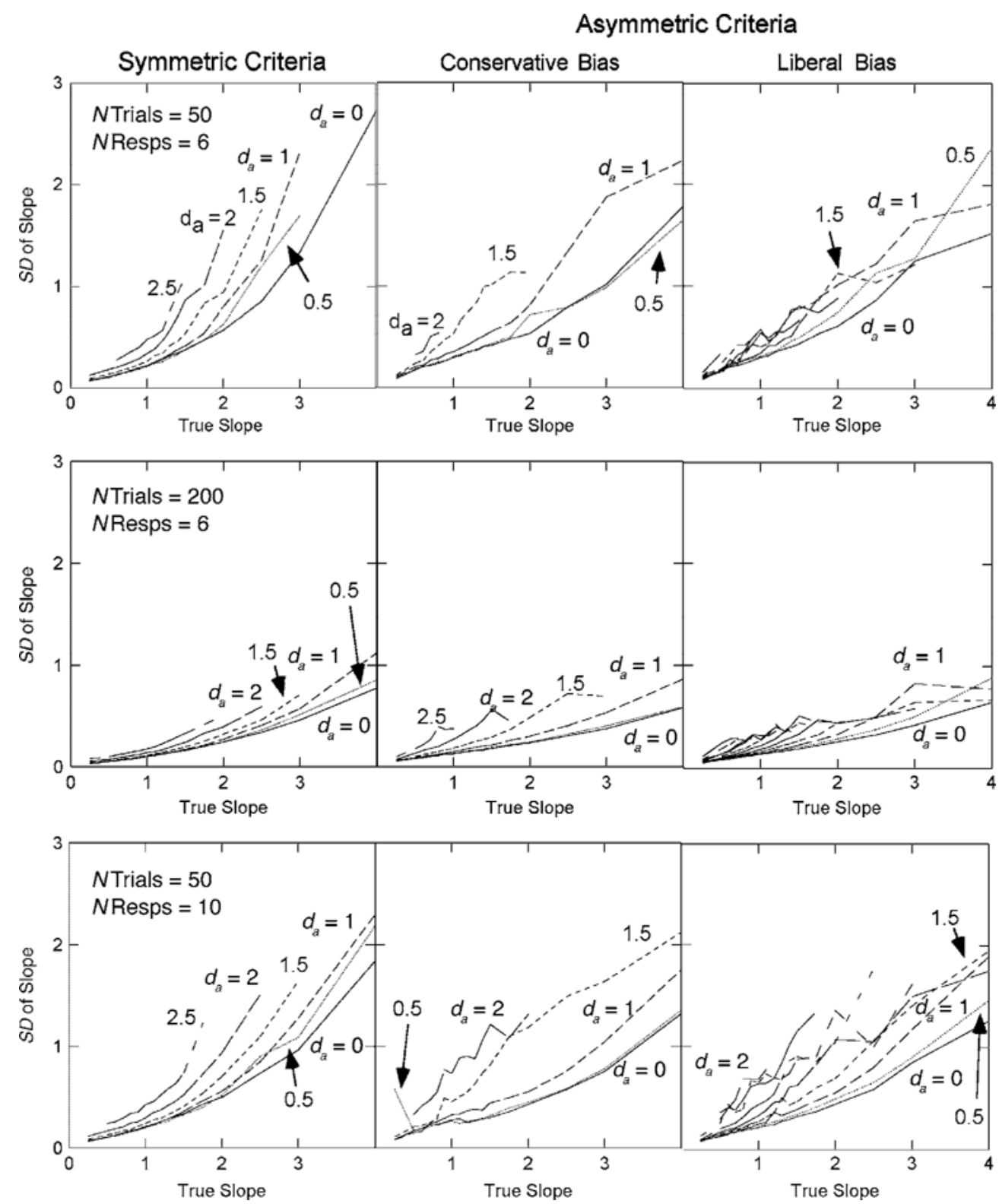

Figure 8. Standard error in estimating $s$, as a function of true $d_{a}$ and true $s$.

pared when based on the same number of trials, and (3) high sensitivity levels ( $d_{a}$ higher than about 2.0) bring with them large standard errors.

\section{Data Analysis}

Area and distance measures of sensitivity. Our calculations support the use of the area measure $A_{z}$ for measuring discrimination performance: It has low bias and a standard error that follows a binomial pattern. There are several different ways to calculate ROC area, and $A_{z}$ is the index of choice if the underlying distributions are approximately normal. For normal representations, it is superior to $A_{g}$, which usually underestimates the true area because of its connect-the-dots algorithm for finding area. (Pollack and Hsieh's [1969] use of this statistic avoids the problem by simulating a continuous rating instrument.) When the underlying distributions are distinctly not normal, $A_{g}$ is preferred. ${ }^{3}$

The distance measure $d_{a}$ has advantages as well; in particular, when combined with $s$, the slope of the $z \mathrm{ROC}$, it provides a good summary of the normal representation. (This advantage is attenuated, however, by the imprecision of estimates of $s$.) It is arguably preferred to $d_{e}^{\prime}$ because of its simple relation to $A_{z}$ (Equation 2).

Estimating confidence intervals. The mean and standard error are the raw materials for building confi- 
Area below lowest criterion $=.05$
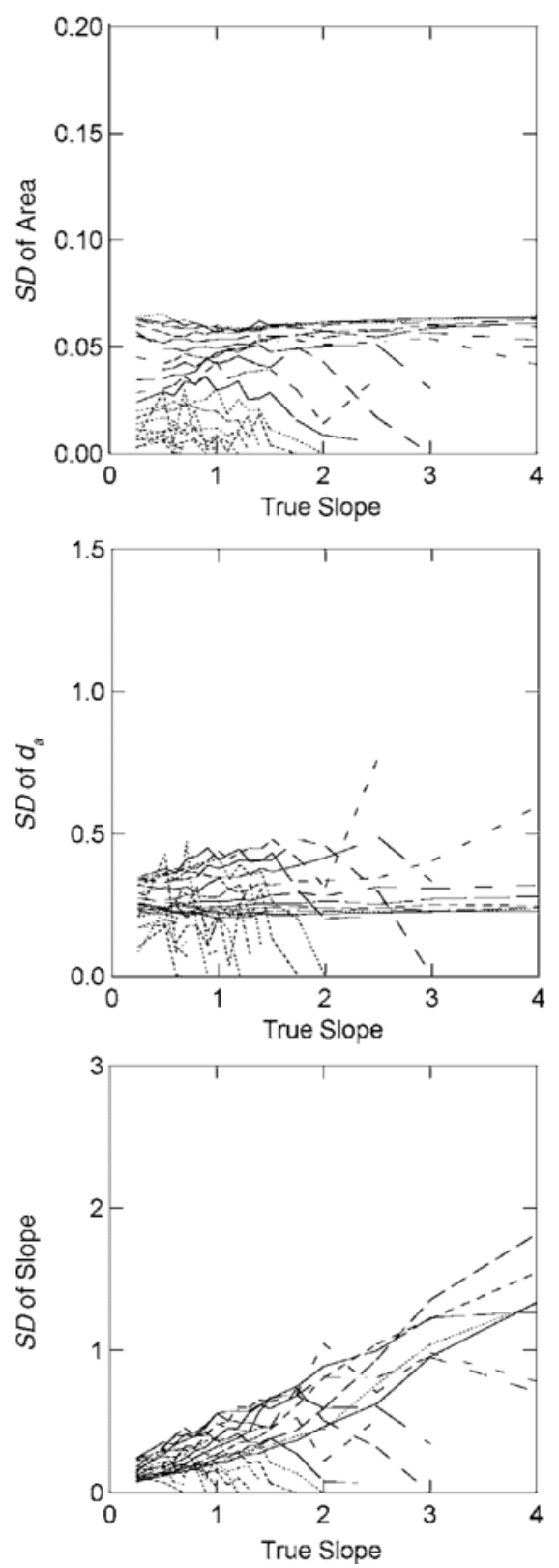

Area below lowest criterion $=25$
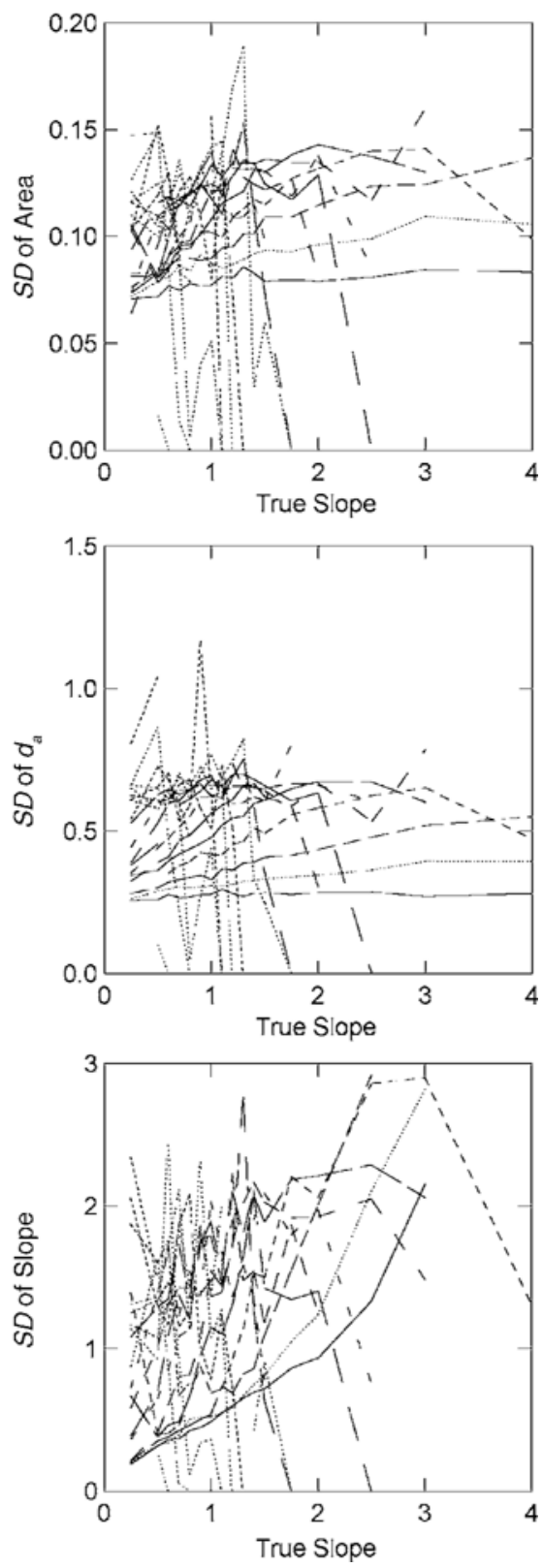

Figure 9. Illustration of the effects of critp on standard errors in the liberal response-bias case. Each row displays one summary statistic, and the columns contrast critp $=.05$ and critp $=.25$. In all cases, $N_{\text {trials }}=50$ and $N_{\text {resps }}=6$. Note that the scale of the panels showing $S D$ of area is not the same as that in Figure 4.

dence intervals and are the only ingredients necessary if the sampling distribution is Gaussian. When a value of a test statistic is observed, the best estimate of the corresponding parameter is the mean of the sampling distribution, and the $95 \%$ confidence interval extends (for normal distributions) 1.96 standard errors above and below it. We illustrate the construction of such intervals for each of our three ROC statistics using the same example:
Suppose that for an experiment in which $N_{\text {trials }}=50$ and $N_{\text {resps }}=4$ we find $s=1.2, d_{a}=1$, and, therefore (by Equation 2 ), $A_{z}=.76$. How can $95 \%$ confidence intervals around these estimates be determined?

Several calculations can be made using the on-line tables. One first examines the locations of the criteria to determine whether they are symmetric, conservative, or liberal, and to estimate the value of critp. Suppose in this 
Table 3

Excerpts From On-Line Tables of Statistical Bias and Standard Error for the Symmetrically Placed Response Criteria $($ critp $=.1)$

\begin{tabular}{|c|c|c|c|c|c|c|c|c|c|}
\hline \multirow[b]{2}{*}{ Statistic } & \multirow[b]{2}{*}{ True $d_{a}$} & \multirow[b]{2}{*}{ True $s$} & \multirow[b]{2}{*}{$N_{\text {trials }}$} & \multicolumn{6}{|c|}{$N_{\text {resps }}$} \\
\hline & & & & 4 & 5 & 6 & 7 & 8 & 10 \\
\hline \multicolumn{10}{|c|}{ Section 1} \\
\hline Average of Bias $s$ & 1 & 1.1 & 50 & 0.0679 & 0.0688 & 0.0367 & 0.0465 & 0.0451 & 0.0384 \\
\hline & & & 200 & 0.0117 & 0.0064 & 0.0139 & 0.0089 & 0.0131 & 0.0050 \\
\hline & & 1.2 & 50 & 0.0889 & 0.0777 & 0.0351 & 0.0523 & 0.0479 & 0.0331 \\
\hline & & & 200 & 0.0247 & 0.0214 & 0.0111 & 0.0137 & 0.0089 & 0.0107 \\
\hline \multicolumn{10}{|c|}{ Section 2} \\
\hline Average of $S D$ Slope & 1 & 1.1 & 50 & 0.2933 & 0.2732 & 0.2349 & 0.2433 & 0.2434 & 0.2244 \\
\hline & & & 200 & 0.1300 & 0.1120 & 0.1116 & 0.1067 & 0.1034 & 0.0992 \\
\hline & & 1.2 & 50 & 0.3484 & 0.3027 & 0.2589 & 0.2890 & 0.2702 & 0.2447 \\
\hline & & & 200 & 0.1559 & 0.1337 & 0.1246 & 0.1159 & 0.1113 & 0.1179 \\
\hline \multicolumn{10}{|c|}{ Section 3} \\
\hline Average of Bias $d_{a}$ & 1 & 1.1 & 50 & 0.0191 & 0.0271 & 0.0269 & 0.0189 & 0.0296 & 0.0250 \\
\hline & & & 200 & 0.0014 & 0.0071 & 0.0009 & 0.0011 & 0.0019 & 0.0051 \\
\hline & & 1.2 & 50 & 0.0028 & 0.0136 & 0.0130 & 0.0208 & 0.0268 & 0.0240 \\
\hline & & & 200 & 0.0033 & 0.0015 & 0.0052 & 0.0102 & 0.0040 & -0.0043 \\
\hline \multicolumn{10}{|c|}{ Section 4} \\
\hline Average of $S D d_{a}$ & 1 & 1.1 & 50 & 0.2389 & 0.2301 & 0.2306 & 0.2275 & 0.2274 & 0.2309 \\
\hline & & & 200 & 0.1173 & 0.1120 & 0.1109 & 0.1142 & 0.1105 & 0.1076 \\
\hline & & 1.2 & 50 & 0.2535 & 0.2343 & 0.2332 & 0.2224 & 0.2245 & 0.2301 \\
\hline & & & 200 & 0.1248 & 0.1164 & 0.1166 & 0.1068 & 0.1092 & 0.1064 \\
\hline \multicolumn{10}{|c|}{ Section 5} \\
\hline Average of Bias Area & 1 & 1.1 & 50 & 0.0010 & 0.0030 & 0.0029 & 0.0013 & 0.0036 & 0.0025 \\
\hline & & & 200 & -0.0004 & 0.0009 & -0.0005 & -0.0005 & -0.0003 & 0.0005 \\
\hline & & 1.2 & 50 & -0.0029 & 0.0000 & -0.0001 & 0.0018 & 0.0031 & 0.0023 \\
\hline & & & 200 & -0.0001 & -0.0004 & 0.0004 & 0.0016 & 0.0002 & -0.0016 \\
\hline \multicolumn{10}{|c|}{ Section 6} \\
\hline Average of $S D$ Area & 1 & 1.1 & 50 & 0.0511 & 0.0495 & 0.0497 & 0.0489 & 0.0486 & 0.0497 \\
\hline & & & 200 & 0.0258 & 0.0244 & 0.0244 & 0.0250 & 0.0240 & 0.0235 \\
\hline & & 1.2 & 50 & 0.0548 & 0.0504 & 0.0500 & 0.0479 & 0.0478 & 0.0496 \\
\hline & & & 200 & 0.0272 & 0.0256 & 0.0255 & 0.0233 & 0.0240 & 0.0234 \\
\hline
\end{tabular}

case the criteria are symmetric and critp $=.1$. An excerpt from the complete table for $N_{\text {trials }}=50$ and $N_{\text {resps }}=$ 4 is shown in Table 3.

It is necessary to start with statistical bias, because the standard error calculations depend on the true values of the parameters, which are more accurately estimated after bias has been taken into account. The bias in $s$ is much greater than that in $d_{a}$ (ranging up to $30 \%$ rather than up to $5 \%$ ), so the starting point is to estimate the true value of $s$. Our starting guess is that $d_{a}=1$ (i.e., no bias). Section 1 of Table 3 shows that if true $s=1.2$, the amount of bias is 0.089 and, thus, observed $s=1.289$, whereas if observed $s=1.1$ the amount of bias is 0.068 and observed $s=1.168$. Interpolation reveals that an observed $s$ of 1.2 corresponds to a true $s$ of 1.13 . Section 2 shows that the interpolated standard error of $s$ for $d_{a}=$ $1, s=1.13$ (using the adjusted value), is 0.2349 . Assuming that the sampling distribution is Gaussian, the $95 \%$ confidence interval is $1.13 \pm(1.96)(0.2349)=$ $1.13 \pm 0.46$. This is a wide interval; the bias could be reduced by a factor of 3 and the interval width by a factor of 2 if the number of trials were quadrupled.
Now consider $d_{a}$. Section 3 of Table 3 indicates a bias of 0.020 if true $s=1.1$ and a bias of 0.003 if true $s=1.2$; interpolating shows that the bias would be 0.015 for true $s=1.13$. Because the bias is small, little error is introduced by assuming that it is constant in a small range, so an observed $d_{a}=1.0$ corresponds to true $d_{a}=1.0-$ $0.0152=0.985$. The standard error, found in Section 4, is 0.2389 , so the confidence interval is $0.985 \pm$ $(1.96)(0.2389)=0.985 \pm 0.47$. For $A_{z}$ there is virtually no bias (Section 5), so .76 is the center of the interval. The standard error is .0510 (Section 6), so the interval is $.76 \pm(1.96)(.0510)=.76 \pm .10$. Because estimates of $d_{a}$ entail more bias than those of $A_{z}$, adjusted values of these two statistics are not exactly related by Equation 2 .

These calculations are based on the assumption that the sampling distributions are Gaussian, but in fact our simulations provide direct information on this issue. For each of the statistics under study, we constructed theoretical 95\% confidence intervals in the manner just described, then counted the percentage of our simulations in which the observed value of the statistic fell either below the lower bound or above the upper bound. For 


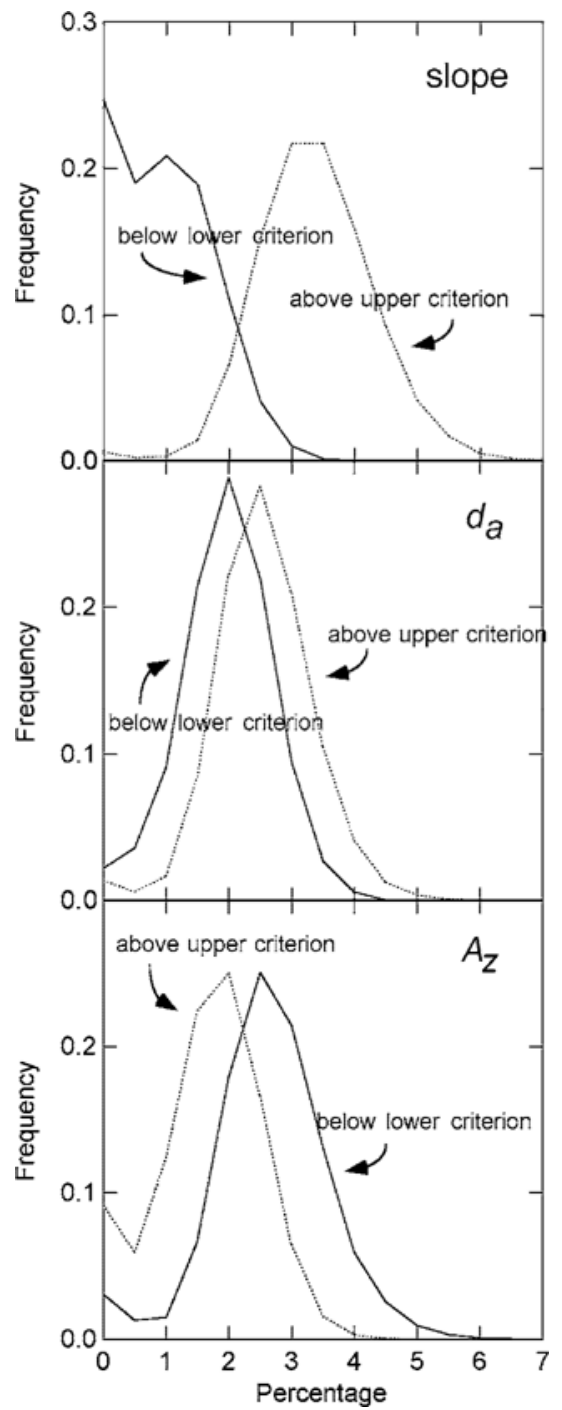

Figure 10. Distributions of the percentage of simulations that fall above the upper limit or below the lower limit of confidence intervals, calculated assuming normal distributions. If the Gaussian assumption were accurate, the distributions would be tightly grouped in the region of $2.5 \%$. The distributions are fairly tight for $A_{z}$ and $d_{a}$ but not for $s$. The distributions also reveal asymmetries in that more outcomes exceed the upper limit than fall below the lower limit for $d_{a}$ and $s$, and more outcomes fall below the lower limit than exceed the upper limit for $A_{z}$.

Gaussian distributions, almost all percentages should be about $2.5 \%$. Figure 10 shows the distributions of these percentages, for the symmetric case only.

The range of the distributions is from $0 \%$ to approximately $6 \%$. For $s$ and $d_{a}$, the percentage in the upper tail is greater than $2.5 \%$ and the percentage in the lower tail is less than $2.5 \%$. For $A_{z}$, the reverse is true. A likely cause of these shifts, and of the evident skew, is that each statistic is bounded, $d_{a}$ and $s$ being bounded below by 0 and $A_{z}$ being bounded above by 1 . The tightness with which the distributions cluster around $2.5 \%$ varies. The percentage of these distributions that is between $1 \%$ and $4 \%$, to take an arbitrary measure, is about $61 \%$ for $s$, $93 \%$ for $d_{a}$, and $87 \%$ for $A_{z}$.

The implication of Figure 10 is that the Gaussian assumption is reasonable for $d_{a}$ and $A_{z}$ but not for $s$. How can a confidence interval of $s$ be constructed? It is not possible to use the figure to "correct" Gaussian confidence intervals, because the figure averages over many quite different parameter values and applies only to the symmetric case. A very conservative reaction to these calculations is to view the large percentages in Figure 10, about $6 \%$, as typical, and thus treat putative $95 \%$ confidence intervals as $88 \%$ intervals $(1-2 \times 6 \%)$. However, a wide range of parameter values enters into the distributions in Figure 10, and, particularly in evaluations of the $z$ ROC slope $s$, choices of these parameters are important. Estimates of $s$ require a larger number of trials than do similarly precise estimates of $d_{a}$ and $A_{z}$, and close spacing of criteria (i.e., high values of critp) increases the variance of the sampling distributions of all three statistics. Large values of $N_{\text {trials }}$ and small values of critp do not contribute much to the tails of the distributions in Figure 10 and make the Gaussian strategy less error prone. If data with nonoptimal values of $N_{\text {trials }}$ and critp are unavoidable, direct estimates of the form of the distribution (and, thus, confidence intervals) can be obtained with bootstrapping or jackknifing techniques (Efron \& Tibshirani, 1993).

Comparing estimates obtained with different numbers of trials. As was noted earlier, the amount of bias in an estimate of these parameters depends on the number of trials on which the estimate is based. This phenomenon (which is most likely to arise in comparisons across experiments) could influence the apparent magnitude of an experimental effect. The problem is most likely to occur for $s$, the statistic with the greatest bias.

Groups of participants. All of these calculations are for data from a single observer. Analyzing individual data is not uncommon in sensory applications and in medical diagnosis (in which differences among X-ray readers, for example, are important), but in other content areas groups of participants are compared and betweenparticipants data are used to estimate the standard error. In such cases, we recommend first correcting each observed slope and sensitivity value for bias, using the online tables, and then subjecting the corrected estimates to standard statistical techniques.

A third possibility, common when few trials can be obtained for any individual (for example, in research with young children or impaired populations), is to pool data across a set of observers before calculating SDT statistics. Our calculations apply somewhat loosely to this situation: Sensitivities and response strategies differ among the individuals being averaged, but it is common to assume that the error thus introduced is modest. For estimates of single ROC points, some support for this assumption has been presented by Macmillan and Kaplan (1985). In fact, Hautus (1997) has shown that pooled $d^{\prime}$ 
(again, for single ROC points) is less biased than average $d^{\prime}$ when the number of trials per subject is low.

\section{Alternative Analyses of ROC Data}

Many researchers collect ROC data and analyze them with detection theory methods, and our calculations provide a useful addition to these methods. The rationale for using SDT, however, varies. In some applications, ideal observer models generate distributions of known shape (Gaussian in some auditory detection situations; Green $\&$ Swets, 1966), and a parametric model is the appropriate framework in which to compare observer performance with this baseline. In other areas, such as recognition memory, normal distributions are generated by hypothesized cognitive processing, and SDT is an integral part of the resulting models (Gillund \& Shiffrin, 1984).

In yet other applications, a detection-theoretic decision process arises, but the underlying distributions are not normal. Visual detection, for example, has long been analyzed in terms of Poisson distributions that arise from the nature of light (Hecht, Schlaer, \& Pirenne, 1942). A recent recognition memory model (DeCarlo, 2002) postulates distributions that are mixtures of two normal distributions (and, therefore, not themselves normal). In both of these examples, deviations from linearity in $z$ ROCs can be detected empirically. The shape of the underlying distributions is not a core assumption of SDT, and there is a long history of distinguishing alternative representations using ROC data.

Some models that contain the elements of detection theory (i.e., underlying distributions and a decision rule based on observations from them) separate sensitivity from bias in ways that are quite different from the standard SDT model. Dynamic modeling, for example (Link, 1992), incorporates speed as well as accuracy; what might appear to be sensitivity changes in a static model may be interpreted as criterion shifts in a dynamic one. Balakrishnan (1999) has offered a reinterpretation of the version of the rating design in which both a binary response and a subsequent rating are required. Using new nonparametric measures, he argues that changes in payoffs and presentation probability do not result in criterion shifts, but instead alter the distributions themselves. Whether this approach is superior to SDT is controversial (Balakrishnan \& MacDonald, 2002; Treisman, 2002).

The value of SDT, and of the current contribution to it, varies across three broad categories of application. The clearest justification is from areas in which parametric distributions arise directly from ideal-observer or other substantive theories. Detection theory statistics also provide convenient summaries in content areas that lack such theories, but in which the type of distinction that SDT draws between sensitivity and bias is plausible. The robustness of SDT statistics is comforting in such applications. For those exploring alternative understandings of discrimination itself, the statistical properties of normal parameters will not be of much interest.

\section{CONCLUSIONS}

Considerable recent attention has been afforded the workhorse SDT statistic $d^{\prime}$. This measure is suitable (and, arguably, ideal) for two-response discrimination experiments, but such experiments have a serious limitation: If the distributions underlying the stimulus classes being discriminated have unequal variance, then estimates of $d^{\prime}$ depend on response bias. Rating experiments solve this problem by generating complete ROC curves, which in most cases can be described well by a sensitivity measure and a slope. The statistical properties of these indices, especially slope, are not well known, and it is this gap that we have attempted to fill here.

Perhaps our most important conclusion is that estimates of $z$ ROC slope face many hazards. They are biased, have high variability, and (especially when based on relatively few trials) have non-Gaussian sampling distributions. Our calculations allow improved evaluation of slope estimates from data, but they also provide guidelines for experimental design that allow investigators to avoid the worst consequences of its frequent misbehavior.

\section{REFERENCES}

BALAKRISHNAN, J. D. (1999). Decision processes in discrimination: Fundamental misconceptions of signal detection theory. Journal of Experimental Psychology: Human Perception \& Performance, 25, 1189-1206.

BALAKRISHNAN, J. D., \& MacDonald, J. A. (2002). Decision criteria do not shift: Reply to Treisman. Psychonomic Bulletin \& Review, 9 , 858-865.

BI, J. (2002). Variance of $d^{\prime}$ for the same-different method. Behavior Research Methods, Instruments, \& Computers, 34, 37-45.

Bi, J., EnNis, D. M., \& O'Mahony, M. (1997). How to estimate and use the variance of $d^{\prime}$ from difference tests. Journal of Sensory Studies, 12, 87-104.

DeCARLo, L. T. (2002). Signal detection theory with finite mixture distributions: Theoretical developments with applications to recognition memory. Psychological Review, 109, 710-721.

Devroye, L. (1986). Non-uniform random variate generation. Berlin: Springer-Verlag.

Dorfman, D. D., \& Alf, E., JR. (1969). Maximum likelihood estimation of parameters of signal detection theory and determination of confidence intervals - Rating method data. Journal of Mathematical Psychology, 6, 487-496.

EFron, B., \& TIBSHIRANI, R. J. (1993). An introduction to the bootstrap. New York: Chapman \& Hall.

Gillund, G., \& Shiffrin, R. M. (1984). A retrieval model for both recognition and recall. Psychological Review, 91, 1-67.

Glanzer, M., Kim, K., Hilford, A., \& Adams, J. K. (1999). Slope of the receiver-operating characteristic in recognition memory. Journal of Experimental Psychology: Learning, Memory, \& Cognition, 25, 500-513.

Gourevitch, V., \& Galanter, E. (1967). A significance test for oneparameter isosensitivity functions. Psychometrika, 32, 25-33.

Green, D. M., \& Swets, J. A. (1966). Signal detection theory and psychophysics. New York: Wiley.

Hautus, M. J. (1997). Calculating estimates of sensitivity from group data: Pooled versus averaged estimators. Behavior Research Methods, Instruments, \& Computers, 29, 556-562.

Hecht, S., Schlaer, S., \& Pirenne, M. H. (1942). Energy, quanta, and vision. Journal of General Physiology, 25, 819-840.

LINK, S. W. (1992). The wave theory of difference and similarity. Hillsdale, NJ: Erlbaum.

Lockhart, R. S., \& Murdock, B. B. (1970). Memory and the theory of signal detection. Psychological Bulletin, 74, 100-109. 
Macmillan, N. A., \& Creelman, C. D. (1991). Detection theory: A user's guide. New York: Cambridge University Press.

Macmillan, N. A., \& Creelman, C. D. (1996). Triangles in ROC space: History and theory of "nonparametric" measures of sensitivity and response bias. Psychonomic Bulletin \& Review, 3, 164-170.

MaCMiLlan, N. A., \& KAPLAN, H. L. (1985). Detection theory analysis of group data: Estimating sensitivity from average hit and falsealarm rates. Psychological Bulletin, 98, 185-199.

Metz, C. (1998). ROCKIT [Computer program]. Available at http://wwwradiology.uchicago.edu/sections/roc/kitguide.

Miller, J. (1996). The sampling distribution of $d^{\prime}$. Perception \& Psychophysics, 58, 65-72.

PoLLACK, I., \& HSIEH, R. (1969). Sampling variability of the area under the ROC-curve and of $d_{e}^{\prime}$. Psychological Bulletin, 71, 161-173.

RatClifF, R., McKoon, G., \& Tindall, M. (1994). Empirical generality of data from recognition memory receiver-operating characteristic functions and implications for the global memory models. Journal of Experimental Psychology: Learning, Memory, \& Cognition, 20, 763-785.

Rotello, C. M., Macmillan, N. A., \& Van Tassel, G. (2000). Recallto-reject in recognition: Evidence from ROC curves. Journal of Memory \& Language, 43, 67-88.

SwETS, J. A. (1986a). Form of empirical ROCs in discrimination and diagnostic tasks. Psychological Bulletin, 99, 181-198.

SwETS, J. A. (1986b). Indices of discrimination or diagnostic accuracy: Their ROCs and implied models. Psychological Bulletin, 99, 100-117.

Swets, J. A., \& PicketT, R. M. (1982). Evaluation of diagnostic systems: Methods from signal detection theory. New York: Academic Press.
Treisman, M. (2002). Is signal detection theory fundamentally flawed? A response to Balakrishnan (1998a, 1998b, 1999). Psychonomic Bulletin \& Review, 9 , 845-857.

\section{NOTES}

1. That the Gaussian model is successful does not mean that other models are not. It has long been recognized that distributions of form other than Gaussian also produce near-linear $z$ ROCs (Lockhart \& Murdock, 1970). Such distributional indeterminacy argues for caution in interpreting ROC data, but it may also explain why so many data sets are consistent with the model and why the use of Gaussian ROC analysis is so widespread.

2. Pollack and Hsieh's (1969) report is unclear in some respects. We assumed that $n$, the number of samples, was the number for both $\mathrm{S}$ and $\mathrm{N}$ trials; this is explicitly stated in some places but is ambiguous in others. In some calculations, the value of $n$ is not indicated at all, and we assumed it equaled 40 . Also, the precision with which the samples were recorded, which affects the number of implied criteria, is not given.

3. Area measures based on a single point do not share the advantages of $A_{z}$ and should not be used. In particular, Macmillan and Creelman (1996) showed that the statistic $A^{\prime}$, often claimed to be nonparametric, entails approximately logistic distributions at low levels of sensitivity and approximately rectangular distributions at high levels.

(Manuscript received April 14, 2003; revision accepted for publication August 14, 2003.) 